\title{
A meta-analysis of randomized controlled trials on levetiracetam in the treatment of pediatric patients with epilepsy
}

This article was published in the following Dove Press journal:

Neuropsychiatric Disease and Treatment

\author{
Lanlan Zhang' \\ Chengzhong Wang' \\ Wei $\mathrm{Li}^{2}$ \\ 'Department of Pediatric Neurology, \\ Yancheng Maternal and Child Health \\ Hospital, ${ }^{2}$ Department of Medical \\ Imaging, Jiangsu Vocational College \\ of Medicine, Yancheng, China
}

Objective: To evaluate clinical efficacy, safety, and tolerability of levetiracetam as mono- or adjunctive therapy in the treatment of children and adolescents with epilepsy.

Materials and methods: We performed a meta-analysis of randomized controlled trials published from January 2007 to December 2016 in the databases Web of Science, Medline, Embase, Cochrane Library, and PubMed, Bing, Baidu, Google Scholar, Chinese National Knowledge Infrastructure (CNKI), and Wanfang Data. All of the studies eligible were compared for the efficacy, safety, and tolerability of levetiracetam with other antiepileptic drugs (AEDs) in epilepsy.

Results: Thirteen randomized controlled trials on a total of 1,013 patients met the inclusion criteria in present study. Compared with other AEDs (oxcarbazepine, valproate, sulthiame, carbamazepine, and placebo), we found that levetiracetam had a comparable seizure-free rate (RR 1.16, 95\% CI 1.03-1.31; $P=0.30$ ). Regarding seizure-frequency reduction $\geq 50 \%$ from baseline, levetiracetam also seemed equivalent to other AEDs (RR 1.08, 95\% CI 1.01-1.16; $P=0.35$ ). In spite of patients treated with levetiracetam having a lower incidence of side effects compared with patients treated with other AEDs (RR 0.90, 95\% CI 0.77-1.06), the difference between them was minute and not statistically significant $(P=0.22)$.

Conclusion: Based on this meta-analysis, it seemed that levetiracetam had comparable effects concerning efficacy, tolerability, and adverse events. Nevertheless, 13 studies were insufficient to draw a conclusion that levetiracetam is effective as mono- and adjunctive therapy for all types of epilepsy syndromes and seizures. Larger-sample and more well-designed trials are needed to justify the widespread use of levetiracetam in the treatment of children and adolescents.

Keywords: levetiracetam, epilepsy, children, antiepileptic drug, RCT, efficacy

\section{Introduction}

It has been estimated that the incidence of epilepsy in all pediatric disease is approximately $0.5 \%-1 \%$, most of which will reduce and achieve remission when children have reached maturity. ${ }^{1}$ The main approach to manage epilepsy is still medication therapy, for the reason that poorly managed seizures can increase family and school burden, leading to poor education and social outcomes. Currently, there is no ideal antiepileptic drug (AED) that can satisfy all the requirements of efficacy, safety, and tolerability, because almost all of the AEDs available can cause short- or long-term side effects or complication and some may be very serious.

Levetiracetam (Keppra, UCB Pharma Sa, Braine-I'Allend, Belgium) is a secondgeneration broad-spectrum AED for both children and adults. Although levetiracetam's exact mechanism of action is not clear, it seems that it differs from other AEDs on
Correspondence: Chengzhong Wang Department of Pediatric Neurology, Yancheng Maternal and Child Health Hospital, 34 West Yulong Road, Yancheng, Jiangsu 224002, China Email leevie@126.com 
structure and function when binding to SV2A, which may play a role in the antiepileptic process. ${ }^{2}$ Nowack et $\mathrm{al}^{3}$ found that by modulating SV2 protein interactions, levetiracetam keeps SV2 and synaptotagmin at the synapse at a normal level, and as a result seizures reduce. With regard to pharmacokinetics, it is rapidly and almost totally absorbed $(>95 \%)$ with maximum concentration occurring at 1.3-5.2 hours. After 24-48 hours of initiation, dose-proportional pharmacokinetics reach a steady state in serum levels. ${ }^{4-6}$ For these characteristics, levetiracetam has become a first-line and one of the most used new-generation AEDs as mono- and adjunctive therapy. ${ }^{7}$

In 2006, levetiracetam was approved as a monotherapy AED, but only in Europe and restricted to adulthood and adolescent from 16 years of age for focal onset seizures with or without secondary generalization. ${ }^{8}$ As monotherapy in younger children, levetiracetam use has increased remarkably in the past two decades, by virtue of its efficacy in both partial and general epilepsy. The primary purpose of the present meta-analysis was to systematically collect, critically evaluate, and synthesize current evidence with respect to the efficacy, safety, and tolerability of levetiracetam as monoor adjunctive therapy for children and adolescents with all types of epilepsy.

\section{Materials and methods}

\section{Identification and eligibility of relevant studies}

The authors conducted an exhaustive literature search of all relevant published articles from January 2007 to December 2016. The Web of Science, Medline, Embase, Cochrane Library, PubMed, Bing, Baidu, Google Scholar databases, Chinese National Knowledge Infrastructure (CNKI) and Wanfang Data databases were used. Search terms were "pediatric", “children", “infant”, “childhood", "juvenile", "adolescent", “seizure", "epilepsy", and "levetiracetam". This meta-analysis was performed in compliance with PRISMA (Preferred Reporting Items for Systematic Reviews and Meta-Analysis). Only articles written in English or Chinese were included, and age of participants was restricted to $<16$ years. Only the most recent studies (earliest publication restricted to 2007) and full-text articles were included in this meta-analysis. Reference lists of these articles were also screened for more information connected to the subject.

\section{Study selection and data extraction}

Selection criteria included in our meta-analysis were randomized controlled trials (RCTs), subjects confined to children and adolescents aged $<16$ years, diagnosis of epilepsy, treated with levetiracetam and compared to other AEDs or placebo, and provision of at least two outcomes of interest from seizure-free rate, seizure-frequency reduction from baseline $(\geq 50 \%)$ and side effects. Exclusion criteria were studies comprising both children and adults that did not give results separately, case reports, retrospective studies, studies written in a language other than English or Chinese, and studies with samples $<30$.

A standardized electric form was designed with Excel (Microsoft Office 2013), and then used to record all the information of interest extracted from each of the 13 RCTs: authors, patient demographics, controlled group interventions, patient/custodian informed consent, country, author affiliations, method of randomization, number, age, and male:female ratio in each group, presence of allocation concealment, length of treatment (including dose changes), duration of follow-up, reasons for discontinuation and numbers, and details of adverse events. For the sake of avoiding accidental mistakes, all three authors wrote down all the data independently, and disagreements among reviewers were resolved through consensus.

\section{Statistical analysis}

Data were processed and analyzed with RevMan 5.3. RRs and 95\% CIs were calculated with fixed-effect or random-effect models (depended on heterogeneity), which meta-analyses usually use to calculate consistency. We recorded numbers lost to follow-up of each treatment group and describe relevant data when the number between randomized and analyzed was inconsistent. We assessed statistical heterogeneity and inconsistency of treatment effects using Cochran's $Q$, $\chi^{2}$ test, and $I^{2}$ statistics. ${ }^{9}$ Significant heterogeneity was set at $\chi^{2}>50 \%$ or $P<0.1$ ( $I^{2}<30 \%$ mild, $30 \% \leq I^{2}<50 \%$, moderate, $I^{2} \geq 50 \%$ substantial). Pooled data using a random-effect model (DerSimonian-Laird method) ${ }^{10}$ when significant heterogeneity was identified; otherwise, a fixed-effects model (Mantel-Haenszel method) ${ }^{11}$ was applied. Publication bias was assessed using Begg' ${ }^{12}$ and Egger' ${ }^{13}$ tests. $P<0.05$ was considered statistically significant, except where otherwise specified.

\section{Results \\ Study selection}

Of the 3,657 initial search results, 2,545 duplicates were removed and 1,083 excluded after review of abstracts or titles. In the remaining 29 prospective studies, 16 were excluded finally for the following reasons: no separate data description for adults and children (six), outcome of interest 


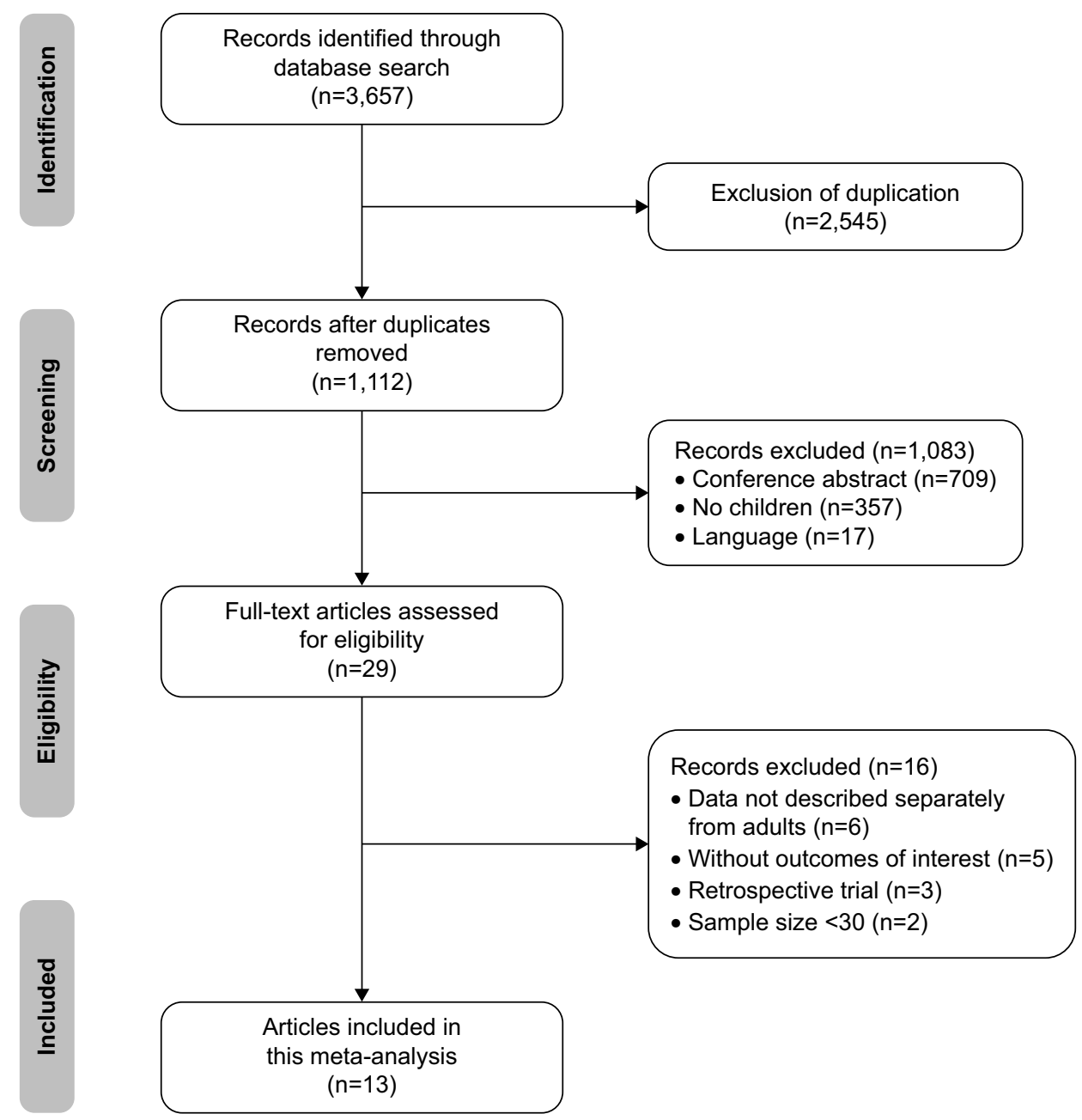

Figure I Study-selection process for this meta-analysis.

not provided (five), and retrospective trials (three). We also excluded two studies due to sample size $<30$ (Figure 1). Finally, $13 \mathrm{RCTs}^{14-26}$ met the criteria and were included in this meta-analysis.

\section{Study characteristics}

The main characteristics of the RCTs are presented in Table 1. All studies were published from 2007 to 2016, whereas only three ${ }^{14,21,25}$ were published before 2010 . The minimum ${ }^{14}$ number of participants in these studies was 39 , the maximum ${ }^{21} 116$. Among the 13 RCTs, two ${ }^{21,25}$ were conducted in the USA, one ${ }^{19}$ in Germany, two ${ }^{14,17}$ in Italy, and eight ${ }^{15,16,18,20,22-24,26}$ in China. With regard to interventions, five $\mathrm{e}^{14,18,23,24,26}$ studies compared levetiracetam with oxcarbazepine, three ${ }^{15,16,20}$ with valproate, three ${ }^{17,21,25}$ with placebo, one ${ }^{19}$ with sulthiame, and one ${ }^{22}$ with carbamazepine. In most trials, the age of patients was 2-16 years; however, in PiñaGarza et al, ${ }^{21}$ the age of participants ranged from 1 month to 4 years, and in Bertsche ${ }^{25}$ the youngest were 6 months old.
All studies included considered body weight, with dosages (levetiracetam) starting at $5-20 \mathrm{mg} / \mathrm{kg} /$ day and increasing until the maximum dosage of 30-60 mg/ $/ \mathrm{kg} /$ day. In spite of 12 months being regarded as the minimum follow-up to draw a conclusion on long-term efficacy, safety, tolerability, and side effects of AED treatment, few trials ${ }^{14,18,26}$ fulfilled this requirement. Nonetheless, two trials ${ }^{19,25}$ continued to follow up to 24 months, but owing to incomplete outcomes were excluded from statistics. Of all studies, four ${ }^{14,19,20,26}$ involved patients with benign childhood epilepsy with centrotemporal spikes (BCECTS), four ${ }^{18,21,22,25}$ partial epilepsy, three ${ }^{16,23,24}$ at least three types of epilepsy or did not report exact epilepsy type, one ${ }^{15}$ partial and general epilepsy but not described separately, and one ${ }^{17}$ was related to absence epilepsy.

\section{Risk-of-bias assessment in individual studies}

Assessment of included studies was conducted according to the Grading of Recommendations Assessment, Development, and Evaluation (GRADE) ${ }^{27}$ criteria, in which all 
Table I Characteristics of RCTs included in this meta-analysis

\begin{tabular}{|c|c|c|c|c|c|c|c|}
\hline Study & Country & $\begin{array}{l}\text { Patients, } \\
\text { n }\end{array}$ & $\begin{array}{l}\text { Age, years } \\
(\text { mean } \pm \text { SD } \\
\text { or mean } \\
{[\text { range] })}\end{array}$ & $\begin{array}{l}\text { Sex } \\
\text { (M/F) }\end{array}$ & Type & $\begin{array}{l}\text { Dose } \\
\text { (mg/kg/day) }\end{array}$ & $\begin{array}{l}\text { Follow-up } \\
\text { (months) }\end{array}$ \\
\hline \multirow[t]{2}{*}{ Chen et $\mathrm{a}^{23}$} & China & Lev 55 & $9.8 \pm 3.5$ & $58 / 45$ & All types & $20-40$ & 6 \\
\hline & & Oxc 48 & & & & $5-40$ & \\
\hline \multirow{2}{*}{ Sun and Zhou ${ }^{15}$} & China & Lev 34 & $7.26 \pm 1.62$ & $20 / 14$ & Focal and & $20-60$ & 6 \\
\hline & & Vpa 34 & $7.19 \pm 1.59$ & $21 / 13$ & general & $15-45$ & \\
\hline \multirow[t]{2}{*}{ Shan ${ }^{26}$} & China & Lev 53 & $8.58(4.4-15)$ & $29 / 24$ & BCECTS & $10-40$ & 12 \\
\hline & & Oxc 51 & $8.45(4.2-14.7)$ & $29 / 22$ & & $10-30$ & \\
\hline \multirow[t]{2}{*}{ Fattore et $\mathrm{a}^{17}$} & Italy & Lev 38 & $8.7 \pm 2.2$ & $15 / 23$ & Absence & $10-30$ & 2 weeks \\
\hline & & Pbo 2I & $7.9 \pm 3.0$ & $12 / 9$ & of epilepsy & $10-30$ & \\
\hline \multirow[t]{2}{*}{ Zhang et $\mathrm{al}^{18}$} & China & Lev 4I & $6.82 \pm 2.79$ & $56 / 52$ & Partial & $5-50$ & 12 \\
\hline & & Oxc 48 & & & & $5-40$ & \\
\hline \multirow[t]{2}{*}{ Liu et al ${ }^{16}$} & China & Lev 50 & $7.6 \pm 2.6$ & ND & All types & $10-40$ & 3 \\
\hline & & Vpa 50 & & & & $5-30$ & \\
\hline \multirow[t]{2}{*}{ Borggraefe et al ${ }^{19}$} & Germany & Lev 21 & $8.7 \pm 1.7$ & $15 / 6$ & BCECTS & $10-30$ & 24 weeks \\
\hline & & Stm 22 & $9.0 \pm 1.5$ & $12 / 10$ & & $2-6$ & \\
\hline \multirow[t]{2}{*}{$\mathrm{Yu}^{20}$} & China & Lev 30 & $8.5 \pm 2.8$ & $37 / 23$ & BCECTS & $20-40$ & 16 weeks \\
\hline & & $V_{p a} 30$ & & & & $10-30$ & \\
\hline \multirow[t]{2}{*}{ Piña-Garza et $\mathrm{a}^{21}$} & USA & Lev 60 & 23.4 months & $30 / 30$ & Partial & $20-50$ & 5 days \\
\hline & & Pbo 56 & 23.5 months & $27 / 29$ & & $20-50$ & \\
\hline \multirow[t]{2}{*}{ Huang and $\mathrm{Zhu}^{22}$} & China & Lev 27 & 6 & $11 / 16$ & Partial & $10-40$ & 6 \\
\hline & & Cbz 26 & 7 & $16 / 10$ & & $5-20$ & \\
\hline \multirow[t]{2}{*}{ Coppola et $\mathrm{al}^{14}$} & Italy & Lev 21 & $10.5(5-13)$ & $11 / 10$ & BCECTS & $5-30$ & 18 \\
\hline & & Oxc 18 & $8.4(3.3-14)$ & $10 / 8$ & & $5-35$ & \\
\hline \multirow[t]{2}{*}{ Hu et $\mathrm{a}^{24}$} & China & Lev 40 & $7.0 \pm 2.1$ & $22 / 18$ & All types & $5-60$ & 6 \\
\hline & & Oxc 4I & $6.8 \pm 1.0$ & $25 / 16$ & & $10-60$ & \\
\hline \multirow[t]{2}{*}{ Levisohn et $\mathrm{al}^{25}$} & USA & Lev 64 & $10.6 \pm 3.5$ & $39 / 25$ & Partial & $20-60$ & 12 weeks \\
\hline & & Pbo 34 & $10.3 \pm 3.7$ & $17 / 17$ & & $20-60$ & \\
\hline
\end{tabular}

Abbreviations: RCTs, randomized controlled trials; Lev, levetiracetam; Oxc, oxcarbazepine; Vpa, valproate; Pbo, placebo; Stm, sulthiame; Cbz, carbamazepine; ND, no data; BCECTS, benign childhood epilepsy with centrotemporal spikes.

assessments of included studies were classified as at low risk of bias, unclear risk of bias, and high risk of bias. For this meta-analysis, there were two ${ }^{21,25}$ studies regarded as at low risk of bias, seven ${ }^{14,16-20,22}$ at unclear risk of bias, and the remaining four ${ }^{15,23,24,26}$ at high risk of bias. Details with regard to risk of bias are provided in Figure 2. A synthesis GRADE profile table (GRADE Pro 3.6) ${ }^{28}$ was used to show evidence profiles of outcomes included in this meta-analysis, evidence quality in terms of seizure-free rate, seizure-frequency reduction $\geq 50 \%$ from baseline, and adverse events. All studies included in this meta-analysis were assessed as being of moderate quality (Table 2).

\section{Synthesis of results}

All studies reported detailed seizure-free rate 100\% and responder rate $\geq 50 \%$, in addition to the adverse-event rate. Pooled estimates from these RCTs suggested that levetiracetam showed no superiority over other AEDs (oxcarbazepine, valproate, sulthiame, carbamazepine, and placebo) on seizurefree rate (RR 1.09, 95\% CI 0.92-1.30; $P=0.30$, Figure 3 ). Likewise, seizure-frequency reduction from baseline $\geq 50 \%$ between levetiracetam and other AEDs looked equivalent (RR 1.05, 95\% CI 0.95-1.15; $P=0.35$, Figure 4). Furthermore, five studies provided more detailed efficacy, in which seizure-frequency reduction from baseline $\geq 75 \%$ (but not include seizure-free) was recorded. In accordance with our analysis, there was no statistically significant difference between levetiracetam and other AEDs regarding this result (RR 0.92, 95\% CI, 0.67-1.25; $P=0.58$ ).

As a result, it seemed that patients treated with levetiracetam experienced equivalent effects compared with patients treated with other AEDs. However, we observed that in three studies ${ }^{17,21,25}$ in which placebo was chosen as intervention, it turned out that levetiracetam did have an advantage (seizure-free, RR 4.26, 95\% CI 1.90-9.45, $P<0.05$; seizurefrequency reduction from baseline $\geq 50 \%$, RR $1.79,95 \% \mathrm{CI}$ $1.26-2.53 ; P<0.05)$.

Two studies ${ }^{17,21}$ used 24-hour or 48-hour electroencephalography other than seizure frequency to evaluate efficacy. There were four studies ${ }^{15,20,25,26}$ that used extra outcome parameters, such as quality of life and recognition functions, as a supplementary-evaluation approach. Levisohn et $\mathrm{al}^{25}$ 


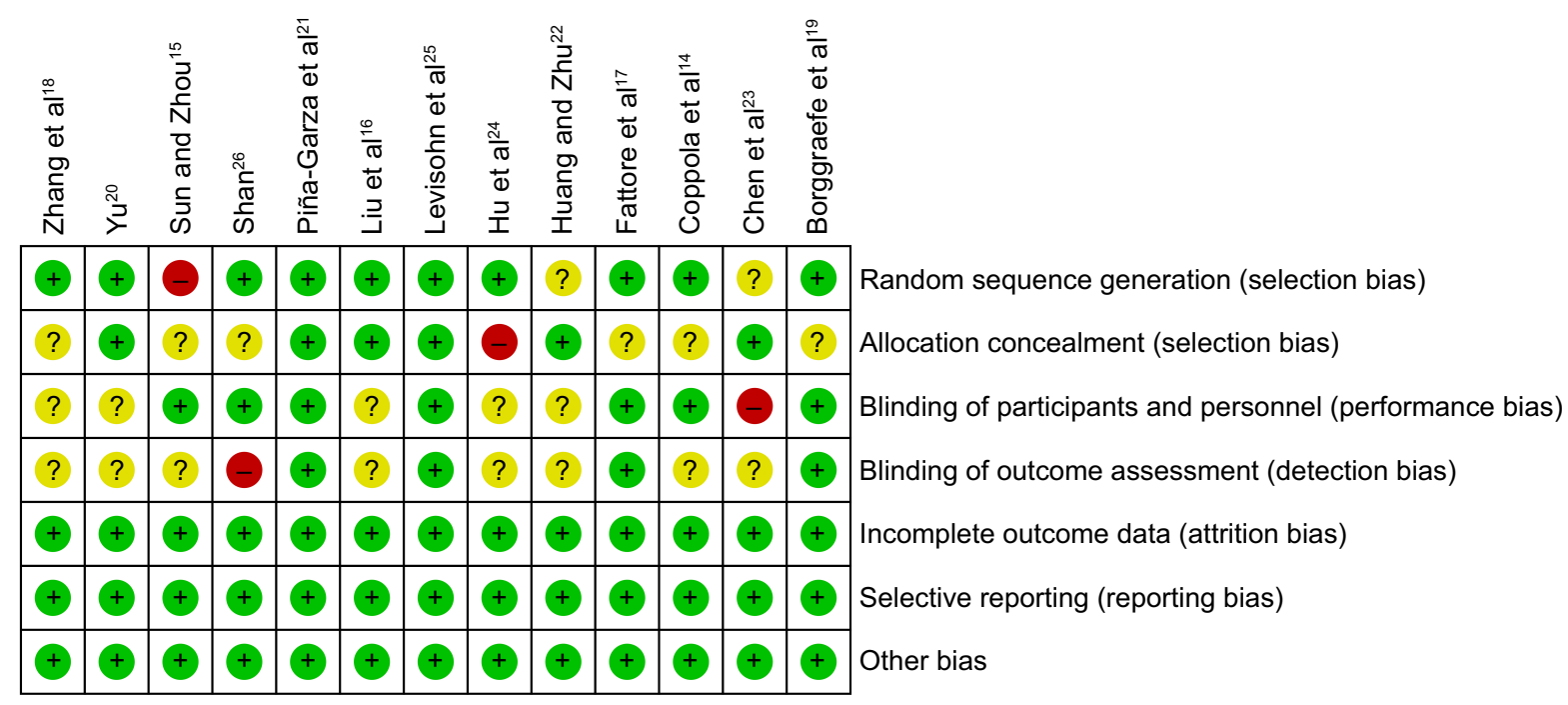

Figure 2 Risk-of-bias summary.

Note: + low risk; $\odot$ high risk; ? unclear risk.

performed a study on neurocognitive effects between children treated with levetiracetam and placebo (64 versus 34) following the Wide Range Assessment of Memory and Learning (second edition) ${ }^{29}$ and the Leiter International Performance Scale - revised attention and memory. ${ }^{30}$ Both tests indicated that there was no statistically significant difference between the two treatment groups $(P=0.202$ and $P=0.9437$, respectively). However, another study conducted by $\mathrm{Yu}^{20}$ involved children treated with levetiracetam or valproate (30 versus 30 ). She evaluated recognition function with verbal intelligence quotient, performance intelligence quotient, full intelligence quotient, and short-term visual memory according to the Wechsler Intelligence Scale for Children. After 3 and 6 months of treatment, she found that scores in children treated with levetiracetam were significantly higher than those treated with valproate. Moreover, the various indices after 6-month treatment were significantly higher than those after 3-month treatment. Differences were statistically significant $(P<0.05)$. Similarly, Sun and Zhou ${ }^{15}$ assessed quality of life of 68 patients treated with levetiracetam and valproate (34 versus 34) according to Quality of Life in Childhood Epilepsy questionnaire, observing that after treatment, life-quality scores in the levetiracetam group $(80.63 \pm 7.02)$ were higher than the valproate group $(63.37 \pm 5.96)$, and differences were statistically significant $(P<0.05)$.

Considering that more than half of the epilepsy types in the RCTs included were partial and BCECTS (four versus four), we conducted a subgroup analysis among them. The results indicated that regarding seizure-free rate and reduction from baseline $\geq 50 \%$, levetiracetam treatment patients in BCECTS group was better than in patients with partial epilepsy, and the difference was statistically significant (seizure-free, RR 2.2, 95\% CI 1.84-2.83, $P<0.05$; reduction $\geq 50 \%$ from baseline, RR $1.51,95 \%$ CI 1.30-1.74, $P<0.05$ ). However, owing to insufficient studies and samples (192 patients in partial group and 125 in BCECTS group), we could not conclude that levetiracetam efficacy in the treatment of patients with BCECTS was superior over partial epilepsy.

All trials reported the occurrence of adverse events, and for the most part adverse events were mild and transient. The most common symptoms were irritability and somnolence, in addition to headache, dizziness, skin rash, and decreased appetite. However, five studies ${ }^{17-19,21,25}$ reported adverse effects so serious enough to terminate levetiracetam treatment. In seven studies ${ }^{15,16,20,22-24,26}$ adverse-event percentages on levetiracetam were lower than other AEDs, and in the other six studies ${ }^{14,17-19,21,25}$ adverse-event percentages on levetiracetam were higher. As a whole, there was no statistically significant difference between levetiracetam and other AEDs (RR 0.90, 95\% CI 0.77-1.06; $P=0.22$, Figure 5).

\section{Discussion}

As a new generation antiepileptic medication, levetiracetam possesses the virtues of a favorable pharmacokinetic profile, novel mechanism, and minimal drug-drug interactions. Based on the 13 RCTs, we found that levetiracetam is an effective adjunctive or monotherapy agent for control of BCECTS and partial and other types of epilepsy. In children, the recommended levetiracetam dose is $10-60 \mathrm{mg} / \mathrm{kg} /$ day, 


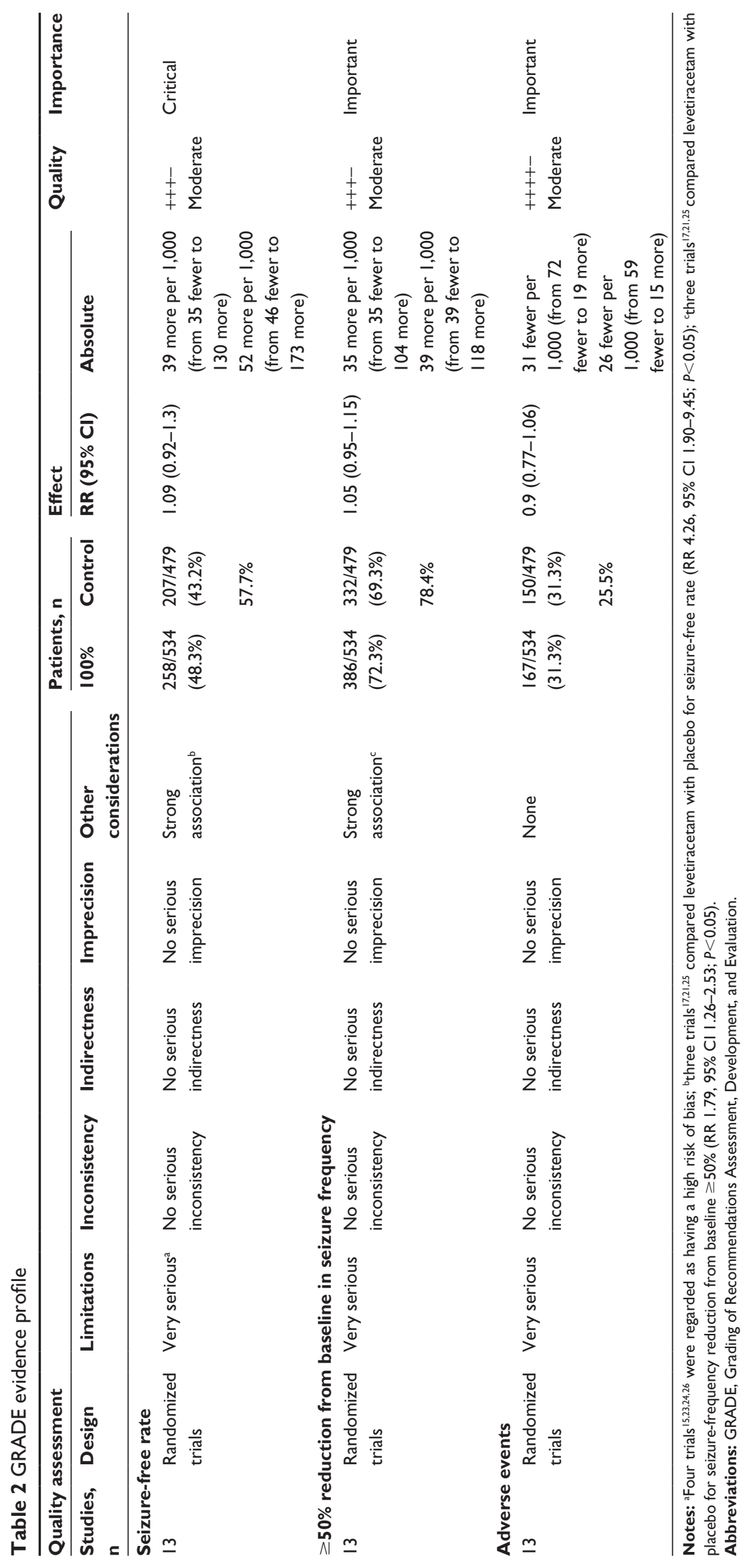




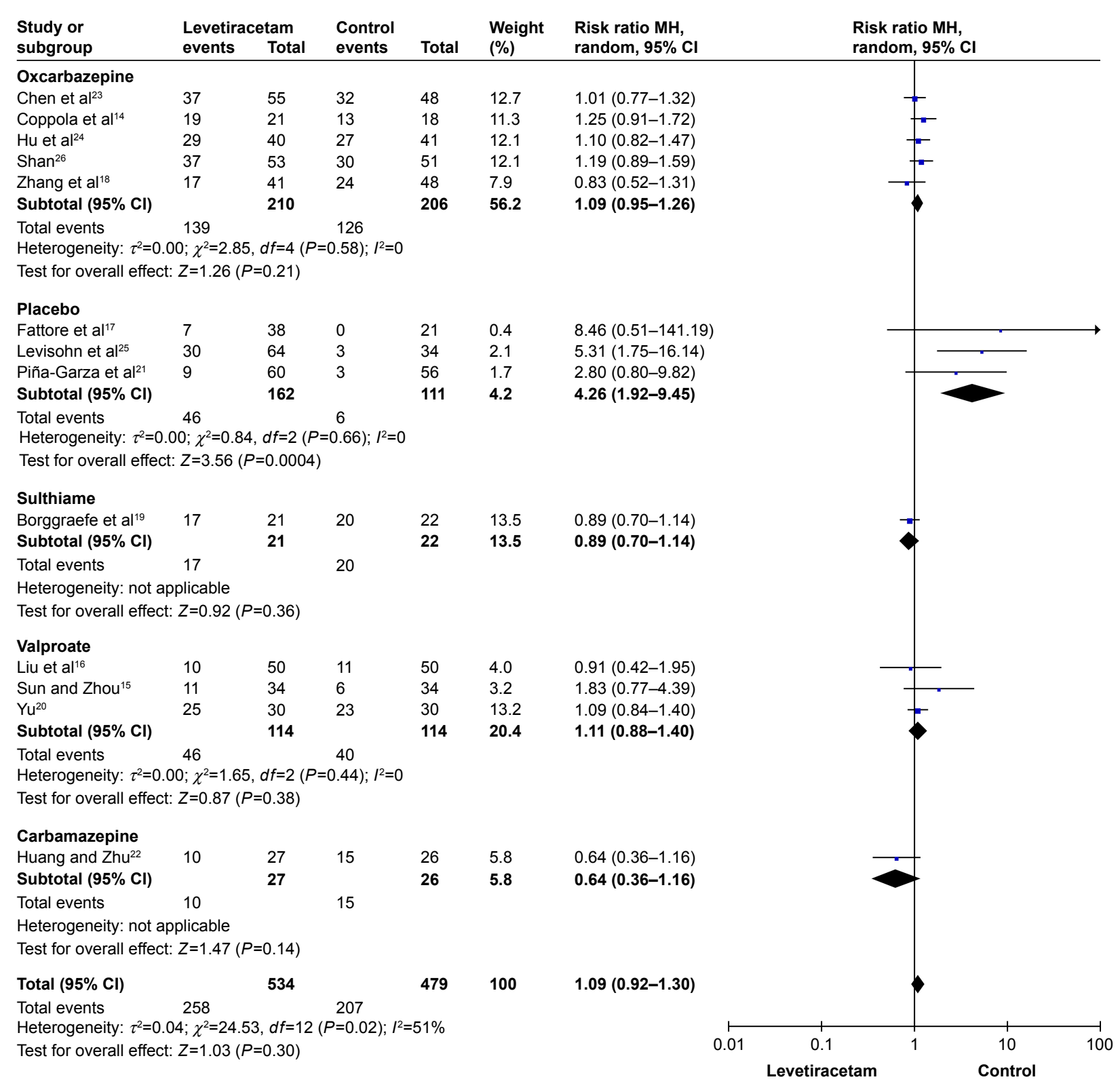

Figure 3 Forest plot of seizure-free rate: levetiracetam versus other AEDs. Abbreviations: MH, Mantel-Haenszel; AEDs, antiepileptic drugs.

which amounts to $1,000-3,000 \mathrm{mg} /$ day in adulthood. The pharmacokinetics of levetiracetam between children and adults are very similar, except that the elimination rate in children is faster. ${ }^{1,31}$ Nevertheless, in accord with our study, it seemed that differences between levetiracetam and other AEDs in respect of seizure-free rate, seizure-frequency reduction from baseline, and occurrence rate of adverse events did not reach statistical significance.

A meta-analysis ${ }^{32}$ and systemic reviews ${ }^{8,33}$ have compared the efficacy, safety, and tolerability of levetiracetam with other AEDs in patients with epilepsy. Lo et $\mathrm{al}^{32}$ included eight studies on levetiracetam as AED for both children and adults with epilepsy. Their investigation suggested that as a monotherapy agent, levetiracetam will play an important role in treating newly diagnosed epilepsy of all types (reduction $\geq 50 \%$, RR $2.15,95 \%$ CI $1.65-2.82$ ) by virtue of its efficacy, safety, and tolerability compared to other AEDs. They also observed that the occurrence rate of adverse events was comparable to other AEDs. In the systemic review conducted by Halma et al, ${ }^{33}$ however, different results were noticed. In that study, they found that behavioral side effects were more frequent in groups treated with levetiracetam compared to other AEDs, but the rate these of behavioral side effects leading to discontinuation of levetiracetam was only $2 \%$. 


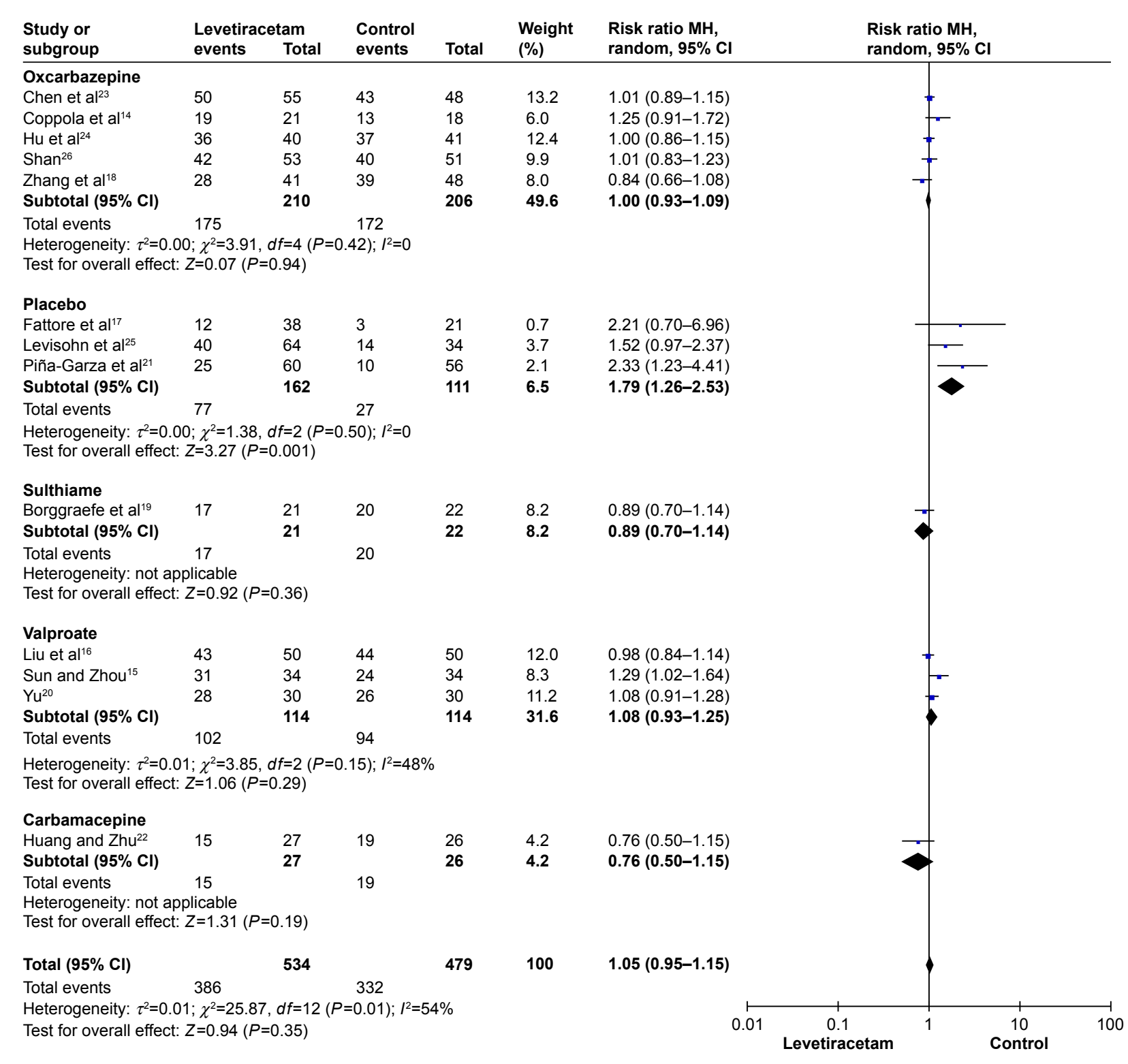

Figure 4 Forest plot of seizure-frequency reduction $\geq 50 \%$ from baseline: levetiracetam versus other AEDs. Abbreviations: $\mathrm{MH}$, Mantel-Haenszel; AEDs, antiepileptic drugs.

Our meta-analysis extended the previous early metaanalysis and systemic reviews to explore better characterization of evidence for levetiracetam in children with all types of epilepsy. Although the two systemic reviews ${ }^{8,33}$ included trials on children, one ${ }^{33}$ emphasized behavioral side effects and did not provide detailed data on efficacy. The other systemic review ${ }^{8}$ included RCTs, case reports, and retrospective studies, and nearly half the trials included participants aged $<20$ years, whereas the lowest number of patients in studies included in our meta-analysis was 39. Finally, the only meta-analysis ${ }^{32}$ published on RCTs on levetiracetam comprised studies involving both adults and children, and did not give detailed description of results separately.
On one hand, based on this meta-analysis, we found that compared to other AEDs, levetiracetam may be a little more effective concerning seizure-free rate or responder rate, which was chosen as the primary end point in practically all studies. In Coppola et al, ${ }^{14}$ children newly diagnosed with BCECTS were divided to levetiracetam or oxcarbazepine groups, the treated with monotherapy of these two AEDs. During the follow-up of 18-24 (mean 18.5) months, they observed that 19 of $21(90.5 \%)$ in the levetiracetam group and 13 of $18(72.2 \%)$ in the oxcarbazepine group were seizurefree. In spite of the seizure-free rate in levetiracetam group being higher than that in the oxcarbazepine group, the difference between them was not statistically significant $(P=0.17)$. 


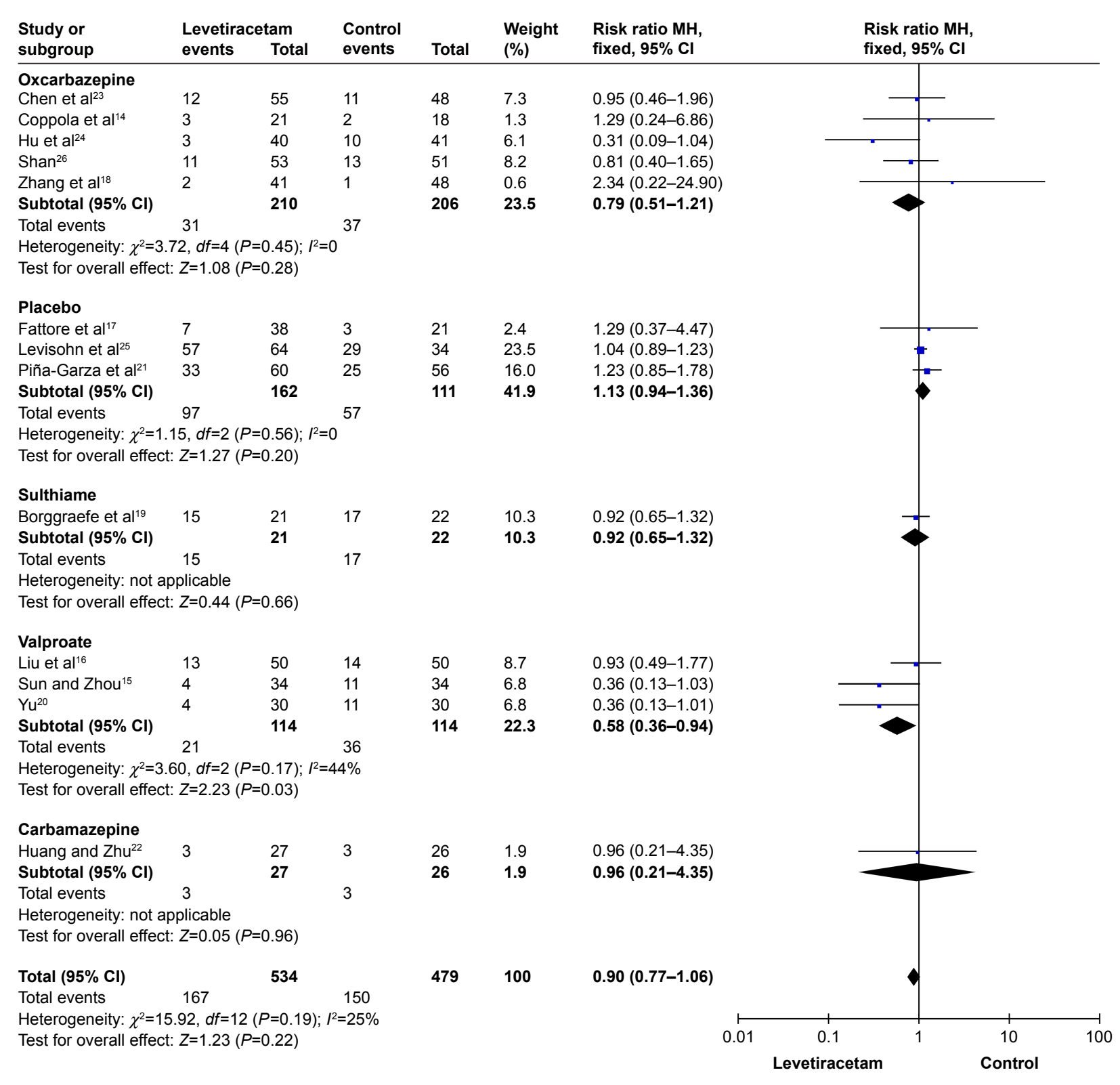

Figure 5 Forest plot of adverse events: levetiracetam versus other AEDs. Abbreviations: $\mathrm{MH}$, Mantel-Haenszel; AEDs, antiepileptic drugs.

Likewise, there were five ${ }^{14,18,23,24,26}$ RCTs included in our studies that compared levetiracetam with oxcarbazepine, and there were no significant differences among them (seizurefree, RR 1.09, 95\% CI 0.95-1.26; $P=0.21$; reduction $\geq 50 \%$ from baseline, RR 1.00, 95\% CI 0.93-1.09; $P=0.94$ ), and similar situations were also found for valproate, sulthiame, and carbamazepine.

On the other hand, in three studies ${ }^{17,21,25}$ that compared levetiracetam with placebo, the difference between the treatments was statistically significant concerning seizure-free rate and seizure-frequency reduction $\geq 50 \%$ from baseline (RR 4.26, 95\% CI 1.92-9.45 $P<0.05$ and RR 1.79, 95\% CI
$1.26-2.53, P<0.05$, respectively). Finally, with regard to safety profile, it seemed that the incidence of adverse events among levetiracetam was also similar to other AEDs. In eight studies, adverse events were mild and transient, and most symptoms disappeared in 4 weeks. Nevertheless, in the other five studies, the adverse events were so serious that a few participants had to terminate treatment and drop out. In Borggraefe et al, ${ }^{19}$ there were nine participants on levetiracetam discontinued due to serious adverse effect compared to three on sulthiame. However, the difference between them was not statistically significant $(23.8 \%$ and $4.5 \%$, respectively). In summary, the tolerability and safety 
of levetiracetam seem to be good, even in very young children ( $<6$ months), ${ }^{21}$ but considering most studies included in this meta-analysis were on BCECTS and partial epilepsy, there is still insufficient data available to confirm that levetiracetam has the same tolerability and safety for other types of seizures and/or epilepsy syndromes.

\section{Limitations}

We tried our best to include more RCTs that met the criteria. However, after exhaustive investigation, it seemed that most were not up to standard. Consequently, there are a few potential limitations in this meta-analysis that should be taken into consideration. Of the included RCTs, the smallest sample 39 , and such a small trial may have led to an overestimation of the treatment effects compared to larger trials. Heterogeneity among the studies was substantial, but considering such details as treatment schedule, variance of AED dosage, quality of trials, and types of epilepsy, all would have influence on the pooled estimates. Results of this meta-analysis are potentially biased for the reason that most studies were from Chinese publications, and readers should bear this in mind, especially with regard to clinical practice. Other limitations were most trials having follow-up $<12$ months, patient self-reporting bias, and different assessment periods.

\section{Conclusion}

As an effective mono- or adjunctive agent, levetiracetam has proved its effectiveness in the treatment of children and adults with epilepsy, and our meta-analysis also supports this point of view, but large-sample, long-term, well-conducted, and double-blind RCTs still need to evaluate its efficacy, safety, tolerability, and adverse effects in children of all age-groups.

\section{Disclosure}

The authors report no conflicts of interest in this work.

\section{References}

1. Shinnar S, Pellock JM. Update on the epidemiology and prognosis of pediatric epilepsy. J Child Neurol. 2002;17 (Suppl 1):S4-S17.

2. Lynch BA, Lambeng N, Nocka K, et al. The synaptic vesicle protein SV2A is the binding site for the antiepileptic drug levetiracetam. Proc Natl Acad Sci U S A. 2004;101(26):9861-9866.

3. Nowack A, Malarkey EB, Yao J, Bleckert A, Hill J, Bajjalieh SM. Levetiracetam reverses synaptic deficits produced by overexpression of SV2A. PLoS One. 2011;6(12):e29560.

4. Patsalos PN. Pharmacokinetic profile of levetiracetam. Pharmacol Ther. 2000;85(2):77-85.

5. Ramael S, Daoust A, Otoul C, et al. Levetiracetam intravenous infusion: a randomized, placebo-controlled safety and pharmacokinetic study. Epilepsia. 2006;47(7):1128-1135.
6. Verrotti A, Cerminara C, Coppola G, et al. Levetiracetam in juvenile myoclonic epilepsy: long-term efficacy in newly diagnosed adolescents. Dev Med Child Neurol. 2008;50(1):29-32.

7. de Smedt T, Raedt R, Vonck K, Boon P. Levetiracetam - part II: the clinical profile of a novel anticonvulsant drug. CNS Drug Rev. 2007; 13(1):57-78.

8. Weijenberg A, Brouwer OF, Callenbach PM. Levetiracetam monotherapy in children with epilepsy: a systematic review. CNS Drugs. 2015; 29(5):371-382.

9. Higgins JPT. Measuring inconsistency in meta-analyses. BMJ. 2003; 327(7414):557-560.

10. DerSimonian R, Laird N. Meta-analysis in clinical trials. Control Clin Trials. 1986;7(3):177-188.

11. Wang D, Zong H, Zhai J, et al. Discussing on [sic] the research of heterogeneity in meta-analysis. Zhongguo Xun Zheng Yi Xue Za Zhi. 2009;9(10):1115-1118.

12. Begg CB, Mazumdar M. Operating characteristics of a rank correlation test for publication bias. Biometrics. 1994;50(4):1088-1101.

13. Egger M, Smith GD, Schneider M, Minder C. Bias in meta-analysis detected by a simple, graphical test. BMJ. 1997;315(7109):629-634.

14. Coppola G, Franzoni E, Verrotti A, et al. Levetiracetam or oxcarbazepine as monotherapy in newly diagnosed benign epilepsy of childhood with centrotemporal spikes (BECTS): an open-label, parallel group trial. Brain Dev. 2007;29(5):281-284.

15. Sun Z, Zhou J. Clinical curative effect and safety of levetiracetam in treating children with epilepsy and the impact of life quality. Zhejiang Med Educ. 2016;15(3):51-53.

16. Liu L, Xiao S, Liu C, et al. The efficacy and safety of valproic acid sodium, topiramate and levetiracetam in treating epilepsy in children. Chin Mod Med. 2016;(22):141-143.

17. Fattore C, Boniver C, Capovilla G, et al. A multicenter, randomized, placebo-controlled trial of levetiracetam in children and adolescents with newly diagnosed absence epilepsy: levetiracetam in absence epilepsy. Epilepsia. 2011;52(4):802-809.

18. Zhang S, Ji C, Zhang J, et al. Clinical effects of levetiracetam and oxcarbazepine for children with partial onset seizures and the impacts on electroencephalogram. Matern Child Health Care China. 2015;(32): $5679-5681$

19. Borggraefe I, Bonfert M, Bast T, et al. Levetiracetam vs. sulthiame in benign epilepsy with centrotemporal spikes in childhood: a doubleblinded, randomized, controlled trial (German HEAD study). Eur $J$ Paediatr Neurol. 2013;17(5):507-514.

20. Yu H. Efficacy and Cognitive effects of Levetiracetam and Sodium Valproate as Monotherapy in Benign Childhood Epilepsy with Centrotemporal Spikes [master's thesis]. Jinan: Shandong University; 2010.

21. Piña-Garza JE, Nordli DR Jr, Rating D, et al. Adjunctive levetiracetam in infants and young children with refractory partial-onset seizures. Epilepsia. 2009;50(5):1141-1149.

22. Huang T, Zhu J. Control study for carbamazepine and levetiracetam in treating children with partial epilepsy as monotherapy. $J$ Pediatr Pharm. 2010;4:25-27.

23. Chen T, Guo Q, Yang Y. Efficacy comparison of levetiracetam and oxcarbazepine monotherapy on children epilepsy. Chongqing Med. 2013; 42(16):1826-1827.

24. Hu D, Mai J, Yang S, Liang H, Li X. Comparative analysis of levetiracetam and oxcarbazepine in treating of children with epilepsy. Chin Mod Dr. 2014;11:34-36.

25. Levisohn PM, Mintz M, Hunter SJ, Yang H, Jones J. Neurocognitive effects of adjunctive levetiracetam in children with partial-onset seizures: a randomized, double-blind, placebo-controlled, noninferiority trial. Epilepsia. 2009;50(11):2377-2389.

26. Shan L. Comparative Study in Treatment of Benign Childhood Epilepsy with Centrotemporal Spikes by Levetiracetam and Oxcarbazepine [master's thesis]. Changchun: Jilin University; 2010.

27. Guyatt GH, Oxman AD, Vist GE, et al. GRADE: an emerging consensus on rating quality of evidence and strength of recommendations. $B M J$. 2008;336(7650):924-926. 
28. Zeng X, Leng W, Li S, Guo Y, Wang P. How to understand and use GRADE system correctly: a briefly outline. Zhongguo Xun Zheng Yi Xue Za Zhi. 2011;9:985-990.

29. Dumont R, Willis JO, Veizel K, Zibulsky J. Wide-Range Assessment of Memory and Learning. 2nd ed. Hoboken, NJ: Wiley; 2014.

30. Roid GH, Miller LJ. Leiter International Performance Scale-Revised. Torrance, CA: WPS; 2002.

31. Glauser TA, Mitchell WG, Weinstock A, et al. Pharmacokinetics of levetiracetam in infants and young children with epilepsy. Epilepsia. 2007;48(6):1117-1122.
32. Lo BWY, Kyu HH, Jichici D, Upton AM, Akl EA, Meade MO. Metaanalysis of randomized trials on first line and adjunctive levetiracetam. Can J Neurol Sci. 2011;38(3):475-486.

33. Halma E, de Louw AJ, Klinkenberg S, Aldenkamp AP, IjffDM, Majoie M. Behavioral side-effects of levetiracetam in children with epilepsy: a systematic review. Seizure. 2014;23(9):685-691.

\section{Publish your work in this journal}

Neuropsychiatric Disease and Treatment is an international, peerreviewed journal of clinical therapeutics and pharmacology focusing on concise rapid reporting of clinical or pre-clinical studies on a range of neuropsychiatric and neurological disorders. This journal is indexed on PubMed Central, the 'PsycINFO' database and CAS, and is the official journal of The International Neuropsychiatric Association (INA). The manuscript management system is completely online and includes a very quick and fair peer-review system, which is all easy to use. Visit http://www.dovepress.com/testimonials.php to read real quotes from published authors.

Submit your manuscript here: http://www.dovepress.com/neuropsychiatric-disease-and-treatment-journal 